(C) The Author(s), 2021. Published by Cambridge University Press on behalf of The Nutrition Society. This is an Open Access article, distributed under the terms of the Creative Commons Attribution licence (http://creativecommons.org/licenses/by/4.0/), which permits unrestricted re-use, distribution, and reproduction in any medium, provided the original work is properly cited.

\title{
Adolescent undernutrition in South Asia: a scoping review
}

\author{
Sara Estecha Querol ${ }^{1,2 *}$ (1) , Paramjit Gill ${ }^{1,2}$, Romaina Iqbal ${ }^{2,3}$, Maartje Kletter ${ }^{1,2}$, Neslihan Ozdemir ${ }^{4}$ and \\ Lena Al-Khudairy ${ }^{5}$ \\ ${ }^{1}$ Academic Unit of Primary Care, University of Warwick, Coventry, UK \\ ${ }^{2}$ NIHR Global Health Research Unit in Improving Health in Slums, University of Warwick, Coventry, UK \\ ${ }^{3}$ Department of Community Health Sciences, Aga Khan University, Karachi, Pakistan \\ ${ }^{4}$ Division of Health Sciences, Bezmialem Vakif University, Istanbul, Turkey \\ ${ }^{5}$ Division of Health Sciences, Warwick Medical School, University of Warwick, Coventry, UK
}

\section{ABSTRACT}

Undernutrition is a growing public health challenge affecting growth and development during adolescence in many low- and middle-income countries. This scoping review maps the evidence on adolescent undernutrition (stunting, thinness and micronutrient deficiencies) in South Asia and highlights gaps in knowledge. Using Arksey and O'Malley's framework and the Joanna Briggs Institute Reviewers' Manual, the search included electronic bibliographic databases (Medline (OVID), Embase, Cochrane Library, Web of Science, CINAHL, PsycInfo, and Scopus) as well as various grey literature sources published up to March 2019. In total, 131 publications met the inclusion criteria of this review. All the included evidence used quantitative data and 115 publications used a cross-sectional design. Nearly $70 \%(n=86)$ of the included publications were conducted in India. Prevalence of undernutrition was reported based on different growth references and cut-offs. Evidence is divided into publications that included an intervention component $(n=12)$ and publications that did not include an intervention component $(n=116)$, and presented in a narrative synthesis. This scoping review provides a wide range of publications on adolescent undernutrition in South Asia and identifies future research priorities in the field.

Key words: Scoping review: adolescence: undernutrition: stunting: thinness: micronutrient deficiency: South Asia (Received 28 October 2020; revised 25 March 2021; accepted 5 April 2021; accepted manuscript published online 16 April 2021)

\section{Introduction}

Adolescents (10-19 years old) represent $1 \cdot 2$ billion individuals worldwide mostly clustered in low-income and middle-income countries $^{(1)}$. Adolescent global health and nutrition have been poorly evaluated ${ }^{(2,3)}$ and health services have struggled to address health needs ${ }^{(4)}$. Adolescence was added in 2016 into the Global Strategy report as an essential component to achieve the Sustainable Development Goals ${ }^{(5)}$. This showed the need to account for adolescents 'because they are central to everything we want to achieve, and to the overall success of the 2030 Agenda, (pg. 5)'. Adolescence is a vulnerable period but also a window of opportunity to address physical and mental health, catch up with growth and ensure improved future outcomes ${ }^{(6)}$. It is also a period of nutritional burden facing undernutrition as well as overnutrition (overweight and obesity) $)^{(4)}$. Lifestyle, eating behaviours, underlying psychosocial factors and socio-economic determinants are key to adolescent health and nutrition ${ }^{(4)}$.

Factors associated with adolescent undernutrition are complex and involve determinants at individual, household and population level $^{(7)}$. The World Health Organization (WHO) identifies some underlying causes of adolescent undernutrition such as diet, diseases, injuries, infections and early pregnancy ${ }^{(8)}$. Undernutrition is assessed by measuring height and weight as well as examining for clinical manifestations and biochemical markers $^{(9)}$. Stunting, thinness and micronutrient deficiencies are forms of adolescent undernutrition ${ }^{(10)}$. Stunting reflects chronic undernutrition, and it is associated with poor cognitive development, lower school performance and reduced economic productivity $^{(11)}$. Stunting is defined as height-for-age 2 SDs below the WHO Child Growth Reference median ${ }^{(12)}$. Thinness or underweight in adolescents is associated with a higher risk of infectious diseases, delayed maturation, reduced muscular strength, work capacity and bone density later in life. In addition, thinness in adolescent girls is associated with adverse pregnancy outcomes and intra-uterine growth retardation ${ }^{(13)}$. Thinness is defined as BMI-for-age 2 SDs below the WHO Growth Reference median ${ }^{(12)}$. Micronutrient deficiencies indicate a lack of essential vitamins and minerals required in small amounts by the body for adequate growth and development ${ }^{(14)}$.

Three hundred and forty million adolescents live in South Asia, accounting for $30 \%$ of the world adolescent population $^{(15)}$. South Asian adolescents are still widely invisible

* Corresponding author: Sara Estecha Querol, email Sara.Estecha-Querol@warwick.ac.uk 
with minimal access to evidence based information and restricted decision-making on issues affecting their life, especially among girls ${ }^{(16)}$. Adolescent girls are more likely to be anaemic, experience gender discrimination, have a poor education and health, and suffer exploitation and violence. The UNICEF reports that $11 \%$ of South Asian adolescent girls aged 15-19 years are stunted, $39 \%$ are thin and $55 \%$ are anaemic ${ }^{(16)}$.

Previous reviews are mainly focused on younger children ${ }^{(17-19)}$ or do not include all forms of adolescent undernutrition ${ }^{(20)}$ in South Asia. A comparison of these studies was published elsewhere ${ }^{(21)}$, revealing the need for a more systematic approach to synthesise the evidence. This scoping review will include a large pool of evidence by following a broad search strategy including all forms of undernutrition as well as including grey literature without restrictions on date of publication. Following the Population, Concept and Context (PCC) strategy ${ }^{(22)}$, we aimed to answer the following question: what do we know about adolescent undernutrition in South Asia from the existing literature? Therefore, this scoping review aims to map the evidence on adolescent undernutrition in South Asia and identify gaps in knowledge.

\section{Methods}

The protocol of this review was previously published ${ }^{(21)}$. The methodology of this scoping review was informed by Arksey and O'Malley's framework ${ }^{(23)}$ and The Joanna Briggs Institute Reviewers' Manual ${ }^{(22)}$. In addition, we followed the Preferred Reporting Items for Systematic reviews and Meta-Analyses extension for Scoping Reviews (PRISMA-ScR) checklist where applicable ${ }^{(24)}$. Since a scoping review involves a methodical integration and presentation of available resources, this study does not require ethics approval.

\subsection{Inclusion and exclusion criteria}

Studies were included if participants were adolescents with a mean age 10-19 years old ${ }^{(25)}$ from Afghanistan, Bangladesh, Bhutan, India, Maldives, Nepal, Pakistan and Sri Lanka ${ }^{(26)}$.

Studies also needed to investigate undernutrition as its primary outcome. Adolescent undernutrition indicators include (1) thinness or underweight (low BMI-for-age), (2) stunting (low height-for-age) and (3) micronutrient deficiencies (lack of essential vitamins and minerals required in small amounts by the body for proper growth and development $\left.{ }^{(14)}\right)$. Studies were included regardless of the growth references or cut-off values followed. Qualitative studies exploring perspectives, experiences or opinions around adolescent undernutrition were included. All study designs of peer-reviewed journals as well as grey literature were included. No restrictions on language or publication date were made. Studies were excluded if they focused on overnutrition indicators alone (obesity and overweight), pregnant or breast-feeding adolescents, adolescent athletes, adolescents with long-term conditions such as diabetes, tuberculosis or HIV, hospitalised adolescents or intervention studies targeting treatment of a specific illness or condition such as diarrhoea.

\subsection{Methods for identifying relevant studies}

The search was conducted using Medline (OVID), Embase, Cochrane, Web of Science, CINAHL, PsycInfo, Scopus, the WHO Library Information System (WHOLIS), eLENA e-Library of Evidence for Nutrition Actions, and Opengrey. The websites of relevant agencies, academic institutions and technical bodies were also searched: WHO, UNICEF, Demographic and Health Surveys (DHS), Program, Planning and Development Department AJ\&K, Global Health Data Exchange (GHDx), World Food Program (WFP) and World Bank eLibrary. Searches were carried out from inception to 11 March 2019. The search strategy included South Asia AND adolescents AND undernutrition. Terms related to undernutrition were also considered in the search strategy. The final search strategy for all databases can be found in the published protocol ${ }^{(21)}$.

\subsection{Selection process and data extraction}

Firstly, titles and abstract were reviewed by two independent reviewers (S.E.Q. and M.K.) following a broad inclusion criterion, that is, studies looking at adolescent undernutrition in South Asia. Papers identified by either or both reviewers were included in the next phase. Secondly, two reviewers independently (S.E.Q. and N.O.) screened full-text studies using the eligibility criteria mentioned above, and reasons for exclusion were documented. At this stage, disagreements were resolved by either discussion or referral to a third reviewer (L.A.K.).

A form was developed and piloted to extract relevant information of the included studies (available in the published proto$\mathrm{Col}^{(21)}$ ). One reviewer (S.E.Q.) extracted data. A second reviewer (L.A.K.) reviewed the results of data charting to resolve any conflict and ensure consistency. For all included studies, key characteristics were charted using an Excel sheet. We extracted data on study characteristics (e.g. author(s)/organisation, year of publication, location, design, and sample size), target population characteristics (e.g. age, sex and setting), adolescent undernutrition indicators (stunting, thinness, and micronutrient deficiencies) and undernutrition outcomes (e.g. prevalence).

We reported the results as a data map presented in a tabular form displaying distribution of publication year, location, characteristics of target population, study design and outcomes. Additionally, a narrative synthesis accompanied the descriptive presentation.

\section{Results}

\subsection{Results of the search}

The search located 6972 records from databases and 1103 records from grey literature such as websites of agencies, academic institutions and technical bodies (Fig. 1). De-duplication resulted in a total of 6181 records that were screened. Title and abstract screening excluded 5640 records. Out of the 541 full-text records assessed for eligibility, 401 did not meet the inclusion criteria. A total of 140 records (131 publications) met the inclusion criteria of this review. Records were grouped under publications if they belonged to the same research project, reporting the group of records as one publication. There were three systematic 


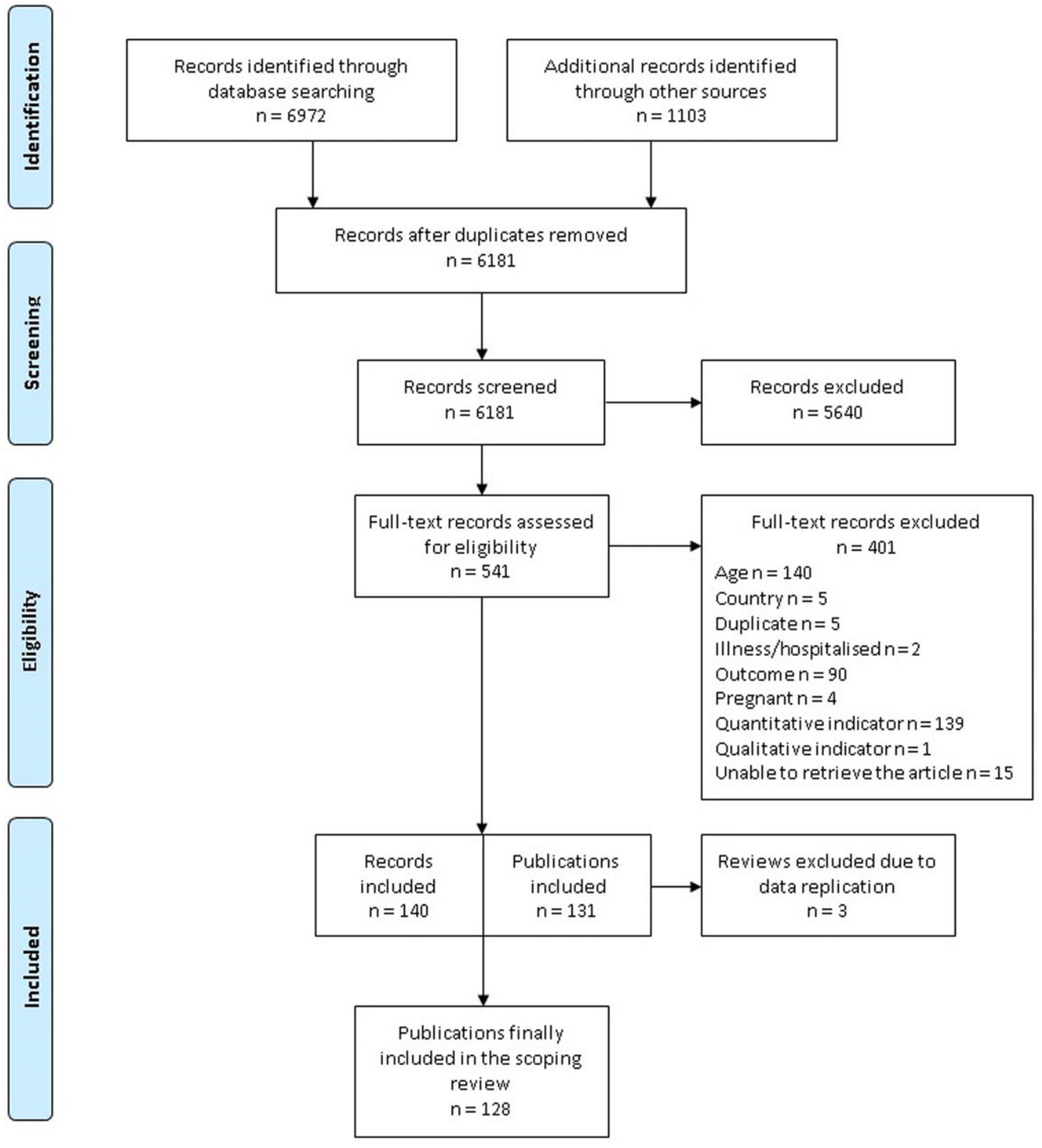

Fig. 1. Selection of sources of evidence in this review using the original PRISMA statement ${ }^{(97)}$.

reviews that were screened for relevant publications. No further evidence was identified from these systematic reviews. Therefore, a total of 128 publications were formally included in this review constituting of 108 original articles, ten letters to editor, six reports from international agencies, three conference abstracts and one communication.

\subsection{Characteristics of the included studies}

Information on the author, year of publication, study title, age and sex of the target population, location, malnutrition indicator(s), classification, growth standards and main findings is provided for the 128 eligible publications (Supplementary material 1). The Supplementary material 1 table categorises the literature into two main groups: one where an intervention component is present $(n=12)$ and one where it is not $(n=116)$.
The majority of the eligible evidence was published in the last 15 years (Table 1 ). The first publication measuring micronutrient deficiencies was in $1975^{(27)}$, the first publication measuring stunting was in $1996^{(28)}$ and the first publication measuring thinness was in $2002^{(29)}$. All included publications reported quantitative outcomes (Table 1). Eligible evidence involved nine randomised controlled trials, two longitudinal studies, one non-randomised prospective trial, one pre-post interventional study and 115 cross-sectional studies (Table 1).

3.2.1. Geographical distribution of the evidence. The majority of publications (67\%) were conducted in India (Table 1). The geographical distribution of the literature was aggregated in some of the states or provinces of Bangladesh, India, Nepal, Pakistan and Sri Lanka (Fig. 2). Evidence from Bhutan, 
Table 1. Characteristics of the included studies

\section{Year of publication}

Before 2000

2000-2004

2005-2009

2010-2014

2015-2019

Type of data

Quantitative

Qualitative or mixed methods

Study design

RCT

Longitudinal studies

Non-randomised prospective trial

Pre-post interventional study

Cross-sectional studies

\section{Country}

Afghanistan

Bangladesh

Bhutan

India

Maldives

Nepal

Pakistan

Sri Lanka

Multinational

Community

Multi-setting (nationally representative)

Rural

Slum

Urban

Urban and rural

Unknown

Setting

Community based

School based

Both

Unknown

Sex

Girls

Boys

Both

Undernutrition indicator

Stunting

Underweight

Micronutrient deficiency

Stunting and underweight

Stunting and micronutrient deficiency

Underweight and micronutrient deficiency

Stunting and underweight and micronutrient deficiency
$\%$ out of 128 publications included
Afhanistan and Maldives was obtained solely from the Global School-based Student Health Survey ${ }^{(30-32)}$. The setting was only reported in 76 publications (60\%) (Table 1), of which 32 were conducted in rural areas (25\%), 8 in slum areas (7\%), 14 in urban areas (11\%), 10 in both urban and rural areas (8\%), and 12 were nationally representative samples (9\%). Sixty-nine of the included publications were school-based (53\%), and 34 were community-based (35\%) (Table 1).

3.2.2. Target population. Sex-stratified analysis was provided in 77 publications (60\%) (Table 1). Forty-five publications reported data on girls only, and six publications included boys only. In terms of participants' age, 82 publications focused exclusively on the age range of 10-19 years. Sixteen publications had a

\begin{tabular}{|c|c|}
\hline 7 & $5 \%$ \\
\hline 5 & $4 \%$ \\
\hline 37 & $29 \%$ \\
\hline 40 & $31 \%$ \\
\hline 39 & $30 \%$ \\
\hline 128 & $100 \%$ \\
\hline 0 & $0 \%$ \\
\hline 9 & $7 \%$ \\
\hline 2 & $2 \%$ \\
\hline 1 & $1 \%$ \\
\hline 1 & $1 \%$ \\
\hline 115 & $90 \%$ \\
\hline 1 & $1 \%$ \\
\hline 17 & $13 \%$ \\
\hline 1 & $1 \%$ \\
\hline 86 & $67 \%$ \\
\hline 1 & $1 \%$ \\
\hline 4 & $3 \%$ \\
\hline 6 & $5 \%$ \\
\hline 9 & $7 \%$ \\
\hline 3 & $2 \%$ \\
\hline 12 & $9 \%$ \\
\hline 32 & $25 \%$ \\
\hline 8 & $7 \%$ \\
\hline 14 & $11 \%$ \\
\hline 10 & $8 \%$ \\
\hline 52 & $40 \%$ \\
\hline 34 & $27 \%$ \\
\hline 69 & $53 \%$ \\
\hline 3 & $2 \%$ \\
\hline 22 & $17 \%$ \\
\hline 45 & $35 \%$ \\
\hline 6 & $5 \%$ \\
\hline 77 & $60 \%$ \\
\hline 16 & $13 \%$ \\
\hline 30 & $23 \%$ \\
\hline 40 & $31 \%$ \\
\hline 27 & $21 \%$ \\
\hline 2 & $2 \%$ \\
\hline 4 & $3 \%$ \\
\hline 9 & $7 \%$ \\
\hline
\end{tabular}

mean age of 10-19 years. In publications where the mean age was greater than 10-19 years, data regarding adolescents were only extractable in 30 publications. The sample size was extracted from 122 publications (95\%). The sample size ranged from 41 to 16245 , with a mean of 1704 adolescents. In six publications, it was not possible to extract the sample size because it was not reported. Adolescents were anaemic at baseline in four publications $^{(33-36)}$.

3.2.3. Undernutrition indicators. Thinness (low BMI-for-age) was frequently ( $n=70$ ) used to measure adolescent undernutrition. Stunting (low height-for-age) was used as an undernutrition indicator in 54 publications, and micronutrient deficiency was also reported in 55 publications (Table 1 ). 
Fig. 2. Geographical distribution of the literature across South Asian countries. The total number does not equal the total number of publications included in the scoping review as some publications focused on more than one country, state or province.

Twenty-seven publications categorised adolescent nutritional status using age- and sex-specific anthropometric measures using National Center for Health Statistics (NCHS) growth charts (Supplementary material 1). Other growth references considered appropriate to assess BMI-for-age or height-forage were WHO reference $2007(n=22)$, Centers for Disease Control and Prevention (CDC) growth charts $(n=11)$, National Health and Nutrition Examination Survery (NHANES) $(n=11)$, WHO reference $1995(n=9)$, and Indian Academy of Pediatrics (IAP) growth charts $(n=5)$. Ten publications used International Obesity Task Force (IOTF) criteria on sex-age-specific BMI cut-offs for thinness, and three publications used Waterlow's classification of stunting. The citations of the listed growth references are provided in Supplementary material 1.

Anaemia was the most reported sign of a micronutrient deficiency (Table 2). It was reported in 38 publications, of which 20 referenced the WHO classification of low haemoglobin concentration. Other micronutrient deficiencies examined were vitamin A $(n=13)$, folic acid $(n=9)$, vitamin B12 $(n=7)$, vitamin $\mathrm{D}(n=6)$, vitamin $\mathrm{C}(n=5)$, zinc $(n=5)$, vitamin B2 $(n=3)$, iodine $(n=3)$ and iron $(n=2)$.

3.2.4. Publications that included an intervention component. Results of publications that include an intervention component $(n=12)$ are presented in Supplementary material 1. A brief on findings of these publications is summarised below.

Among the publications that include an intervention component, supplementation was the most prevalent type of intervention, using food fortification in six publications and capsules in five. In addition, there was one school feeding program which provided daily balanced meals to a cohort of tribal students attending a school in rural south India ${ }^{(37)}$. The authors of this publication classified the students according to attendance: 'new' students less than 1 year and 'old' students more than 1 year of attendance. Comparison of thinness prevalence in these groups showed that $50 \%$ of 'new' and $37.5 \%$ of 'old' students were thin; however, the difference was not significantly different between the two groups.

In Sri Lanka, iron and zinc capsules consumed daily on school days for 24 weeks showed improvements in the prevalence of anaemia and zinc deficiency but did not decrease the rate of stunting among adolescents ${ }^{(38)}$. Various publications compared the efficacy of different micronutrient supplementation combinations among anaemic adolescents ${ }^{(33-35)}$ and vitamin $\mathrm{D}$ deficient adolescents ${ }^{(39)}$. A sample of 178 anaemic adolescent schoolgirls in Bangladesh received iron-folic acid - $30 \mathrm{mg} F$ and $400 \mu \mathrm{g}$ folic acid - tablets or multiple micronutrients (MMN) tablets (15 micronutrients, including iron and folic 
Table 2. Micronutrient deficiencies and indicators reported

\begin{tabular}{lc}
\hline Micronutrient & Number of publications \\
\hline Anaemia & \\
Low haemoglobin & 34 \\
No indicator reported & 4 \\
Vitamin A deficiency & \\
Clinical signs (i.e. Bitot's spots) & 5 \\
Low serum retinol & 7 \\
Low plasma retinol & 1 \\
Folic acid deficiency & \\
Low erythrocyte folate & 4 \\
Low serum folic acid & 5 \\
Vitamin B12 deficiency & \\
Low serum vitamin B12 & 7 \\
Vitamin D deficiency & \\
Low serum 25(OH)D & \\
Vitamin C deficiency & 6 \\
Low plasma vitamin C & \\
Zinc deficiency & 5 \\
Low serum zinc & \\
Low plasma zinc & 3 \\
No indicator reported & Vitamin B2 deficiency \\
High EGRAC & \\
lodine deficiency & Low urinary iodine \\
Iron deficiency & 1 \\
Low serum ferritin & 1 \\
\hline
\end{tabular}

acid) ${ }^{(35)}$. The findings showed that even though the effect of iron-folic acid tablets and MMN tablets on reducing anaemia and iron deficiency was similar, MMN supplementation demonstrated a significant enhancement of other micronutrients' status. The team examined the relative efficacy of long-term ( 52 weeks) once-weekly and twice-weekly MMN supplementation ${ }^{(34)}$. Both supplementation frequencies were equally efficacious in decreasing anaemia; however, twice-weekly MMN was more efficacious than once-weekly MMN in improving vitamin A, riboflavin and folic acid status. Another randomised controlled trial studied the effect of non-iron containing ayurvedic preparations to treat nutritional anaemia in adolescent students in India ${ }^{(33)}$. The authors found that a daily dose of two non-iron containing ayurvedic preparations improved anaemia in the study participants. An intervention providing $1500 \mu \mathrm{g} /$ week doses of vitamin D to vitamin D deficient adolescents revealed that 4-, 6- and 8week supplementation regimens were equally efficacious in achieving vitamin D sufficiency ${ }^{(39)}$. Micronutrient-fortified biscuits confirmed to be effective in improving not only micronutrient deficiencies but also thinness and stunting among Indian adolescent girls ${ }^{(40)}$. Evidence showed that food fortification is a cost-effective and sustainable strategy to improve micronutrient deficiencies among adolescents in Bangladesh ${ }^{(41)}$ and India ${ }^{(42)}$, respectively. School-based interventions showed that vitamin D fortification $^{(43)}$ and iron fortification ${ }^{(44)}$ was an effective strategy for wider use in school feeding programs. School-based supplementation programs also resulted in higher adherence due to supervision ${ }^{(45)}$. However, a project providing weekly iron-folic acid tablets to adolescent girls did not find any difference in the impact on anaemia prevalence between school girls (supervised) and non-school girls (unsupervised) ${ }^{(42)}$.
3.2.5. Publications that did not include an intervention component. We identified 116 publications that assessed nutritional status, that is, the prevalence of undernutrition. Among these, six publications compared stunting and/or thinness prevalence using different growth references, and 38 publications assessed undernutrition risk factors such as age, sex, demographic determinants and socio-economic status. Results of publications that did not include an intervention component are displayed in Supplementary material 1, and a narrative summary is provided below.

The prevalence of stunting, thinness and micronutrient deficiencies was challenging to compare because the included publications followed different growth references. Prevalence varied widely and is presented in Supplementary material 1. Prevalence of stunting ranged from $7 \%{ }^{(46)}$ to $90 \%{ }^{(47)}$, and prevalence of thinness varied from $1 \cdot 3 \%^{(30)}$ to $63 \%^{(48)}$. Some publications determined prevalence of stunting and/or thinness with different growth references ${ }^{(46,49-53)}$. On the one hand, the prevalence of nutritional status varied greatly with the growth reference employed. Agrahar-Murugkar (2005) showed that stunting prevalence in Indian schoolgirls was 46\% using Waterlow's classification and $7 \%$ using $\leq 2$ SD from median of CDC $2000^{(46)}$. On the other hand, variability of thinness was found to be smaller by Garg et al. (2013), ranging from $6 \cdot 6 \%$ (using $<5$ th percentile of IAP reference) to $17 \%$ (using $\leq 2 \mathrm{SD}$ from median of $\mathrm{CDC}$ 2000) among adolescent boys in India ${ }^{(49)}$. Four publications comparing different references reported that nutritional status prevalence measured by IAP reference was lower compared with prevalence measured by other growth references such as CDC, WHO and $\mathrm{NCHS}^{(49-52)}$.

Sex, age, socio-economic, demographic, dietary and other associated factors were evaluated in relation to stunting and thinness among South Asian adolescents. Four publications reported that stunting in adolescent girls was more common than in adolescent boys $^{(54-57)}$, but the difference was statistically significant in only one publication $^{(55)}$. In contrast, prevalence of stunting was found to be significantly higher among boys than girls in Northeast India, resulting in 1.55 times greater risk of being stunted for boys as compared with girls ${ }^{(58)}$. Prevalence of thinness was found to be higher among boys than girls in eight publications ${ }^{(54,57,59-64)}$ reporting significance in five ${ }^{(54,59-61,64)}$. Overall prevalence of thinness was slightly higher among girls than boys in two publications from India, but there was no sex-specific significant difference ${ }^{(48,65)}$. Indian adolescents showed a significantly higher prevalence of stunting with increased age ${ }^{(55,58)}$. The results of these two publications also showed that the risk of stunting in late adolescence was approximately two ${ }^{(58)}$ to four times ${ }^{(55)}$ higher than in early adolescence. Prevalence of thinness was greater among early adolescents compared with older counterparts ${ }^{(55,59,61,66,67)}$. However, age-specific trends in thinness prevalence were absent in six publications ${ }^{(48,54,64,65,68,69)}$. While stunting was more common amongst adolescents residing in rural areas in India ${ }^{(56,57,70,71)}$, thinness was found to be more prevalent in both urban ${ }^{(70)}$ and rural settings ${ }^{(57)}$. Low socio-economic status was significantly associated with stunting ${ }^{(55,58)}$ and thinness ${ }^{(55,72)}$ among South Asian adolescents. Thinness was significantly higher in adolescent girls living in households with unimproved water sources $^{(67,72)}$. Parents' education ${ }^{(58,63,68)}$, birth order and family size $^{(29,55,69)}$ as well as dietary determinants such as energy 
intake ${ }^{(66,73)}$, micronutrient intake $\mathrm{e}^{(67,73)}$, vegetarianism ${ }^{(66)}$, dietary diversity $^{(72)}$ and food availability ${ }^{(72)}$ were found to be significantly contributing to adolescent undernutrition.

Anaemia (low haemoglobin concentration) ranged between $1 \%{ }^{(74)}$ and $98 \cdot 3 \%{ }^{(75)}$. Prevalence of micronutrient deficiency varied (Supplementary material 1); vitamin A deficiency from $0 \cdot 3 \%{ }^{(76)}$ to $65 \cdot 4 \%{ }^{(77)}$, folic acid deficiency from $1 \cdot 5 \%^{(78)}$ to $54 \cdot 6 \%{ }^{(79)}$, vitamin B12 deficiency from $0 \cdot 44 \%{ }^{(80)}$ to $68 \cdot 3 \%^{(81)}$, vitamin D deficiency from $70 \%{ }^{(82)}$ to $96 \cdot 3 \%{ }^{(83)}$, vitamin $\mathrm{C}$ deficiency from $2 \%^{(36)}$ to $10 \cdot 8 \%^{(77)}$ and zinc deficiency from $28 \cdot 8 \%^{(84)}$ to $72 \cdot 4 \%{ }^{(77)}$. Prevalence of iodine deficiency was $23 \cdot 6 \%{ }^{(85)}$ and $38 \cdot 4 \%{ }^{(86)}$, prevalence of vitamin B2 deficiency was $89 \%^{(36)}$ and prevalence of iron deficiency was $55 \%{ }^{(81)}$.

Prevalence of micronutrient deficiencies and associated factors was studied among South Asian adolescents. Association between the prevalence of vitamin A deficiency and sex was not significant in two publications ${ }^{(76,87)}$; however, another publication found that xerophthalmia as defined by Bitot spots and/or night blindness was significantly associated with adolescent boys ${ }^{(88)}$. While iron deficiency $^{(74)}$ and vitamin D deficiency ${ }^{(82,83)}$ were significantly higher among females, vitamin B12 deficiency was significantly more common among males ${ }^{(89)}$. On age-wise categorisation, two publications did not find a significant difference in prevalence of anaemia among Indian adolescent girls ${ }^{(90,91)}$. The increase in age with the increase in the prevalence of vitamin A deficiency ${ }^{(87)}$ and iron deficiency $^{(74)}$ was found to be statistically significant. Vitamin B12 deficiency $^{(89)}$ and anaemia ${ }^{(91)}$ were significantly higher among rural adolescents compared with their urban counterparts. Ethnicity ${ }^{(74)}$, occupation of the father ${ }^{(92)}$ and education of the mother ${ }^{(93)}$ were associated with anaemia. When categorised on the basis of working status, working adolescent girls from Sri Lanka were at nearly twice the risk of having folic acid deficiency and twice the risk of having zinc deficiency when compared with non-working adolescents ${ }^{(84)}$.

\section{Discussion}

There are limited reviews on adolescent undernutrition in South Asia ${ }^{(17-20)}$. To the authors' knowledge, this study is the first scoping review mapping the available evidence. The search strategy involved seven electronic databases and grey literature. From 6181 records matching the search, 128 met the selection criteria and were therefore included in this scoping review.

The first aim of the present scoping review was to map the evidence on all forms of adolescent undernutrition in South Asia. Several characteristics of the included publications were examined. The evidence was considerably recent as there were only 12 publications from before 2004 . There was available literature on adolescent micronutrient deficiencies since the 1970s; however, the oldest evidence calculating BMI-for-age and height-for-age was published after WHO guidance (1995) on measuring and classifying thinness and stunting in adolescents. Evidence included in this scoping review comprises exclusively quantitative methods employing mostly cross-sectional study design. Literature was clustered in certain states or provinces of some South Asian countries; most publications were conducted in India. Rural areas were the most reported communities. These findings show an uneven geographical distribution of the literature across countries and within countries in South Asia. Half of the publications were school-based, while 35\% were community-based. Despite the large numbers of out-ofschool adolescents in South $\mathrm{Asia}^{(94)}$, recruiting participants from madrassahs, schools and high schools was possibly more accessible than a household survey or door-to-door approach. In this scoping review, we identified that undernutrition was more studied among adolescent girls than boys, and thinness was more studied than stunting or micronutrient deficiencies. In addition, anaemia was the most evaluated micronutrient deficiency.

This scoping review found only 12 publications that included an intervention component, one of which was a school feeding program and the rest were supplementation programs. The age limitation in this scoping review might have excluded some interventions targeting children from birth to adolescence. It is worth noting that we did not assess the effectiveness of these programs in reducing adolescent undernutrition as this falls outside the aims of our scoping review. However, all publications were successful in improving at least one form of adolescent undernutrition. Recommendations from these publications can be inferred. The authors of the school feeding program ${ }^{(37)}$ suggested that noting the exact length of attendance time to the program and quantifying meal intake by each student could be key to examining more accurately the nutritional benefits of school meals and developing detailed nutritional school programs. Recommendations resulting from the supplementation programs highlighted the need to explore long-term efficacy, dose and intake frequency of supplements to enhance adolescent micronutrient status.

A considerable number of publications that did not include an intervention component were available. These publications assessed the prevalence of all forms of adolescent undernutrition in South Asia. Undernutrition prevalence varied greatly. This may be due to the usage of different growth references, classifications and cut-offs as well as differences in characteristics of the target population such as age range and setting. Hence, comparison across publications cannot be made. It is important to note that this scoping review did not assess the quality of undernutrition indicators measured in the included publications; consequently, prevalence could have been overestimated or underestimated. Six publications nevertheless assessed and compared prevalence of stunting and/or thinness using different growth references ${ }^{(46,49-53)}$. The review findings showed that prevalence depended substantially on the growth reference used. The lowest thinness and stunting rates were given by IAP reference ${ }^{(49-52)}$. Applicability of different growth references to Indian schoolchildren was recently examined ${ }^{(95)}$. Similarly to our findings, Singh et al. (2020) observed that IAP reference classified fewer children as stunted and thin than WHO reference 2007. The authors suggested using IAP reference over WHO reference 2007 to evaluate growth in Indian schoolchildren. Further research on applicability of different growth references to South Asian adolescents should be conducted, to enable undernutrition prevalence to be compared across South Asian countries and worldwide.

Undernutrition-associated factors were evaluated in 38 of the included publications in this scoping review. The findings were presented with raw prevalence, $P$ values and odds ratios, 
impeding comparison across publications. In addition, the findings were inconsistent for some of the associated factors. For instance, stunting was found to be more prevalent among girls, but evidence also suggested boys were at greater risk of being stunted. The findings on thinness were slightly more dependable, with boys being thinner than girls. Sex association varied greatly with the micronutrient deficiency studied. Stunting, thinness and micronutrient deficiencies were also associated with age, rural and urban areas, socio-economic status, sanitation, parents' education and occupation, birth order, individual working status, family size and dietary determinants.

The second aim of this scoping review was to identify gaps in knowledge. We found that much of the available literature covers prevalence of adolescent undernutrition in the South Asian context using a cross-sectional design. This indicates that additional research on undernutrition using other study designs is needed to explore causality during adolescence instead of association. In addition, this scoping review did not locate any qualitative or mixed-methods publications. These approaches could contribute to better understanding adolescent undernutrition as well as evaluating if experiences from adolescents match with undernutrition-associated factors given by quantitative research. This scoping review also identified that there is a lack of adolescent undernutrition literature in Afghanistan, Bangladesh, Bhutan, Maldives, Nepal, Pakistan and Sri Lanka, as nearly $70 \%$ of the evidence included was conducted in India. Moreover, we observed that some publications were less systematic than others in reporting method and target characteristics such as age of the sample, community, setting or growth reference. As a consequence, this information was difficult to extract, and so it is missing. Reporting the community (rural, slum, urban or nationally representative sample) is a clear example, as we could not identify it in $40 \%$ of the publications. We noticed greater evidence scarcity from slums and national representative samples as well as research targeting out-of-school adolescents. This review suggests that supplementation and school feeding program research on South Asian adolescents seldom evaluated cost-effectiveness and impact on undernutrition rates in the long term on a large scale. Therefore, future research should aim to be implemented at a national level, also incorporating other types of interventions such as nutrition promotion, weight gain treatment, regulatory interventions (i.e. marketing) or health-related actions (i.e. deworming) to tackle adolescent undernutrition. This scoping review found that thinness (BMI-for-age) was more widely used to measure adolescent nutritional status than stunting (height-for-age). However, both indicators should be calculated since they assess different forms of undernutrition. Finally, existing literature did not appropriately determine whether some undernutrition-associated factors such as sex or age are risk or protective determinants. In addition, little is known about other factors such as dietary intake or sanitation in relation to adolescent undernutrition in South Asia.

Our scoping review has some limitations. Although our search strategy and selection process followed systematic methods, the challenges of searching for grey literature might have obstructed the inclusion of some relevant unpublished evidence $^{(96)}$. Due to time constraints and the large number of included publications, we did not search additional resources by hand searching the evidence reference list. Despite these limitations, we are confident that this scoping review provides a wide perspective on adolescent undernutrition in South Asia.

\section{Conclusion}

This scoping review located a broad range of publications on adolescent undernutrition in South Asia and identified noteworthy gaps in the evidence. Further exploration of additional methods to evaluate adolescent undernutrition in South Asia and its associated factors is necessary to implement effective actions for improving adolescent nutritional status.

\section{Financial support}

This work was supported by NIHR grant number 16/136/87. The research was commissioned by the National Institute of Health Research using Official Development Assistance (ODA) funding. The views expressed are those of the author(s) and not necessarily those of the NHS, the NIHR or the Department of Health and Social Care.

\section{Conflict of interest}

None.

\section{Authorship}

S.E.Q. conceived the idea and developed the methods. P.G., R.I. and L.A.K. contributed substantially to the conception and design. S.E.Q. conducted the searches, retrieved articles and screened evidence. M.K. and N.O. screened evidence. S.E.Q. conducted the analysis and interpretation of data. S.E.Q. wrote the first draft of the manuscript, and P.G. and L.A.K. supported the drafting and editing of the manuscript. S.E.Q., P.G., M.K. and N..O. revised the final manuscript. All authors approved the final manuscript.

\section{Supplementary material}

To view supplementary material for this article, please visit https://doi.org/10.1017/S0954422421000068

\section{References}

1. Lansford JE, Banati P (2018) Handbook of adolescent development research and its impact on global policy. New York: Oxford University Press.

2. Christian P, Smith ER (2018) Adolescent undernutrition: global burden, physiology, and nutritional risks. Ann Nutr Metab $\mathbf{7 2}$, 316-328.

3. Mokdad AH, Forouzanfar MH, Daoud F et al. (2016) Global burden of diseases, injuries, and risk factors for young people's health during 1990-2013: a systematic analysis for the Global Burden of Disease Study 2013. Lancet 387, 2383-2401. 
4. World Health Organization (2005) Nutrition in adolescenceissues and challenges for the bealth sector. Geneva: World Health Organization.

5. World Health Organization (2015) The global strategy for women's, children's and adolescents' bealth Geneva: World Health Organization.

6. Banati P, Camilletti E (2018) Three windows of opportunity using science to inform programming for adolescents and young people. https://blogs.unicef.org/evidence-for-action/ three-windows-of-opportunity-for-adolescents/\#_ftn2 (accessed 15 April 2020)

7. Patton GC, Sawyer SM, Santelli JS et al. (2016) Our future: a Lancet commission on adolescent health and wellbeing. Lancet 387, 2423-2478.

8. World Health Organization (2018) Guideline: implementing effective actions for improving adolescent nutrition. Geneva: World Health Organization.

9. United Nations Children's Fund (2013) Improving child nutrition: the achievable imperative for global progress. New York, USA: United Nations Children's Fund.

10. World Health Organization (2016) What is malnutrition? http:// www.who.int/features/qa/malnutrition/en/ (accessed 19 November 2018)

11. Cashin K, Oot L (2018) Guide to anthropometry: a practical tool for program planners, managers, and implementers. Washington, DC: Food and Nutrition Technical Assistance III Project (FANTA).

12. de Onis M, Onyango AW, Borghi E et al. (2007) Development of a WHO growth reference for school-aged children and adolescents. Bull World Health Organ 85, 660-667.

13. Food and Agriculture Organization, International Fund for Agricultural Development, United Nations Children's Fund et al. (2018) The state of food security and nutrition in the world 2018. Building climate resilience for food security and nutrition. Rome: Food and Agriculture Organization.

14. Ritchie H, Roser M (2020) Micronutrient deficiency. https:// ourworldindata.org/micronutrient-deficiency (accessed 14 April 2020)

15. United Nations Children's Fund (2019) Adolescents in South Asia. https://www.unicef.org/rosa/what-we-do/adolescents (accessed 25 April 2019)

16. United Nations Children's Fund (2019) Adolescent and women's nutrition. https://www.unicef.org/rosa/what-we-do/nutrition/ adolescent-and-womens-nutrition (accessed 25 April 2019)

17. Khan AA, Bano N, Salam A (2007) Child malnutrition in South Asia: a comparative perspective. SAS 14, 129-145.

18. Akhtar S (2016) Malnutrition in South Asia - a critical reappraisal. Crit Rev Food Sci Nutr 56, 2320-2330.

19. Pasricha S-R, Biggs B-A (2010) Undernutrition among children in South and South-East Asia. J Paediatr Child Health 46, 497503.

20. Mak KK, Tan SH (2012) Underweight problems in Asian children and adolescents. Eur J Pediatr 171, 779-785.

21. Estecha Querol S, Al-Khudairy L, Iqbal R et al. (2020) Adolescent undernutrition in South Asia: a scoping review protocol. BMJ Open 10, e031955.

22. Peters M, Godfrey C, McInerney P et al. (2015) The Joanna Briggs Institute reviewers' manual 2015: methodology for JBI scoping reviews. Adelaide, SA Australia: The Joanna Briggs Institute.

23. Arksey H, O'Malley L (2005) Scoping studies: towards a methodological framework. Int J Soc Res Methodol 8, 19-32.

24. Tricco AC, Lillie E, Zarin W et al. (2018) PRISMA extension for scoping reviews (PRISMA-ScR): checklist and explanation. Ann Intern Med 169, 467-473.
25. World Health Organization (2019) Adolescent health and development. http://www.searo.who.int/entity/child_adolescent/ topics/adolescent_health/en/ (accessed 1st February 2019)

26. The World Bank (2019) The World Bank in South Asia. http:// www.worldbank.org/en/region/sar/overview (accessed 28 February 2019)

27. Tandon BN, Ramachandran K, Nath LM et al. (1975) Vitamin A nutritional status of rural community of Khol block in Haryana, North India. A collaborative study. Am J Clin Nutr 28, 14361442.

28. PanterBrick C, Todd A, Baker R. (1996) Growth status of homeless Nepali boys: do they differ from rural and urban controls? Soc Sci Med 43, 441-451.

29. Venkaiah K, Damayanti K, Nayak MU, Vijayaraghavan K (2002) Diet and nutritional status of rural adolescents in India. Eur J Clin Nutr 56, 1119-1125.

30. World Health Organization (2016) Global School-based Student Health Survey: Bhutan 2016 Fact Sheet.

31. World Health Organization (2014) Global School-based Student Health Survey: Afghanistan 2016 Fact Sheet.

32. World Health Organization (2014) Global School-based Student Health Survey: Maldives 2016 Fact Sheet.<cont>

33. Prakash VP, Prakash S, Sharma R, Pal SK. (2010) Sustainable effect of Ayurvedic formulations in the treatment of nutritional anemia in adolescent students. J Altern Complement Med 16, 205-211.

34. Ahmed F, Khan MR, Akhtaruzzaman M et al. (2010) Long-term intermittent multiple micronutrient supplementation enhances hemoglobin and micronutrient status more than iron + folic acid supplementation in Bangladeshi rural adolescent girls with nutritional anemia. J Nutr 140, 1879-1886.

35. Ahmed F, Rahman Khan M, Akhtaruzzaman M et al. (2005) Efficacy of twice-weekly multiple micronutrient supplementation for improving the hemoglobin and micronutrient status of anemic adolescent schoolgirls in Bangladesh. Am J Clin Nutr 82, 829-835.

36. Ahmed F, Khan MR, Banu CP, Qazi MR, Akhtaruzzaman M (2008) The coexistence of other micronutrient deficiencies in anaemic adolescent schoolgirls in rural Bangladesh. Eur $J$ Clin Nutr 62, 365-372.

37. Thomas R, Srinivasan R, Sudarshan H. (2013) Nutritional status of tribal children and adolescents in rural south India: the effect of an NGO delivered nutritional programme. Indian J Pediatr 80, 821-825.

38. Hettiarachchi M, Liyanage C, Wickremasinghe R, Hilmers DC, Abrams SA (2008) The efficacy of micronutrient supplementation in reducing the prevalence of anaemia and deficiencies of zinc and iron among adolescents in Sri Lanka. Eur J Clin Nutr 62, 856-865.

39. Garg MK, Marwaha RK, Khadgawat R et al. (2013) Efficacy of vitamin D loading doses on serum 25-hydroxy vitamin D levels in school going adolescents: an open label non-randomized prospective trial. J Pediatr Endocrinol Metab 26, 515-523.

40. Goyle A (2012) Effect of micronutrient fortified biscuit supplementation on the weight, height and BMI of adolescent girls. Coll Antropol 36, 573-579.

41. Rahman AS, Ahmed T, Ahmed F, Alam MS, Wahed MA, Sack DA (2015) Double-blind cluster randomised controlled trial of wheat flour chapatti fortified with micronutrients on the status of vitamin $\mathrm{A}$ and iron in school-aged children in rural Bangladesh. Matern Child Nutr 11 Suppl 4, 120-131.

42. Vir SC, Singh N, Nigam AK, Jain R (2008) Weekly iron and folic acid supplementation with counseling reduces anemia in adolescent girls: a large-scale effectiveness study in Uttar Pradesh, India. Food Nutr Bull 29, 186-194. 
43. Khadgawat R, Marwaha RK, Garg MK et al. (2013) Impact of vitamin D fortified milk supplementation on vitamin D status of healthy school children aged 10-14 years. Osteoporos Int 24, 2335-2343.

44. Muthayya S, Thankachan P, Hirve S et al. (2012) Iron Fortification of Whole Wheat flour reduces iron deficiency and iron deficiency anemia and increases body iron stores in indian school-aged children. J Nutr 142, 1997-2003.

45. Hyder SM, Haseen F, Khan M et al. (2007) A multiple-micronutrient-fortified beverage affects hemoglobin, iron, and vitamin A status and growth in adolescent girls in rural Bangladesh. $J$ Nutr 137, 2147-2153.

46. Agrahar-Murugkar D (2005) Nutritional status of Khasi schoolgirls in Meghalaya. Nutrition 21, 425-431.

47. Durrani NUR, Ahmad Z, Abbas KA (2007) Nutritional and health status of school age children in Islamabad Capital Territory. Pak Pediatr J 31, 190-194.

48. Bharthi K, Ghritlahre M, Das S, Bose K (2017) Nutritional status among children and adolescents aged 6-18 years of Kolam tribe of Andhra Pradesh, India. Anthropol Rev 80, 153-163.

49. Garg P, Kaur S, Gupta D et al. (2013) Variability of thinness and its relation to cardio-metabolic risk factors using four body mass index references in school-children from Delhi, India. Indian Pediatr 50, 1025-1032.

50. Mandot S, Mandot D, Sonesh JK (2009) Nutritional status of tribal (Garasia) school children of Sirohi district, Rajasthan. Indian Pediatr 46, 437-438.

51. Medhi GK, Hazarika NC, Mahanta J. (2007) Nutritional status of adolescents among tea garden workers. Indian J Pediatr $\mathbf{7 4}$, 343-347.

52. Prashant K, Shaw C (2009) Nutritional status of adolescent girls from an urban slum area in South India. Indian J Pediatr $\mathbf{7 6}$, 501-504.

53. Sikdar M (2012) Prevalence of malnutrition among the Mising children of Northeast India: A comparison between four different sets of criteria. North Am J Med Sci 4, 305-309.

54. Mondal N, Sen J (2010) Prevalence of stunting and thinness among rural adolescents of Darjeeling district, West Bengal, India. Ital J Public Health 7, 54-61.

55. Pal A, Pari AK, Sinha A, Dhara PC (2017) Prevalence of undernutrition and associated factors: a cross-sectional study among rural adolescents in West Bengal, India. Int J Pediatr Adolesc Med 4, 9-18.

56. Rao KM, Balakrishna N, Laxmaiah A, Venkaiah K, Brahmam GN (2006) Diet and nutritional status of adolescent tribal population in nine states of India. Asia Pac J Clin Nutr 15, 64-71.

57. Vashist BM, Jyoti, Goel MK (2009) Nutritional status of adolescents in rural and urban Rohtak, Haryana. Health Popul Perspect Issues 32, 190-197.

58. Rengma MS, Bose K, Mondal N (2016) Socio-economic and demographic correlates of stunting among adolescents of Assam, North-east India. Anthropol Rev 79, 409-425.

59. Bose K, Bisai S (2008) Prevalence of underweight and stunting among school children in West Bengal. Indian J Pediatr $\mathbf{7 5}$, 1272 .

60. Chakrabarty S, Bharati P (2008) Physical growth and nutritional status of the Shabar tribal adolescents of Orissa, India: a cross-sectional study. Malays J Nutr 14, 101-112.

61. Das P, Ray SK, Joardar GK, Dasgupta S (2007) Nutritional profiles of adolescents in a rural community of Hooghly district in West Bengal. Indian J Public Health 51, 120-121.

62. Johnson AR, Balasubramanya B, Jaimol SR, Shaiby SR (2015) Body image perception and nutritional status of adolescents in a school in rural South India. J Indian Assoc Child Adolesc Ment Health 11, 260-278.
63. Khan AB, Nicola B, Khan SR (2013) Double burden of overweight and underweight among school children of Dhaka city, Bangladesh. Obes Res Clin Pract 1, 26.

64. Mondal N (2014) Thinness as major underlying problem among adolescents of northeast India. J Nepal Paediatr Soc 34, 39-47.

65. Mondal N, Terangpi M (2014) Prevalence of undernutrition among tribal adolescents of Karbi Anglong district of Assam, Northeast India. SriLanka J Child Health 43, 154-158.

66. Ameer SR, Ahmad SR, Chandrasekhar A. (2018) Assessment of underweight and its determinants among school going adolescents in Hyderabad. Indian J Public Health Res Dev 9, 77-82.

67. Radhika MS, Swetha B, Kumar BN, Krishna NB, Laxmaiah A. (2018) Dietary and nondietary determinants of nutritional status among adolescent girls and adult women in India. Ann NY Acad Sci 1416, 5-17.

68. Das DK, Biswas R (2005) Nutritional status of adolescent girls in a rural area of North 24 Parganas district, West Bengal. Indian J Public Health 49, 18-21.

69. Debnath M, Tigga PL, Mondal N, Sen J. (2016) Birth order, father's occupation and family size are strongly associated with thinness among bengalee adolescent girls of Darjeeling district, West Bengal (India). J Nepal Paediatr Soc 36, 115-120.

70. Choudhary S, Khichar S, Dabi D et al. (2016) Urban rural comparison of anthropometry and menarcheal status of adolescent school going girls of Jodhpur, Rajasthan, India.J Clin Diagn Res 10, SC8-SC12.

71. Maiti S, Ali KM, De D, Bera TK, Ghosh D, Paul S (2011) A comparative study on nutritional status of urban and rural early adolescent school girls of West Bengal, India. J Nepal Paediatr Soc 31, 169-174.

72. Niranjala AMS, Gunawardena NS (2011) Nutritional status of adolescent females in estates in Haliela, Sri Lanka. Infant Child Adolesc Nutr 3, 260-267.

73. Hettiarachchi M, Liyanage C, Wickremasinghe R, Hilmers D, Abrams S (2006) Nutrient intake and growth of adolescents in southern Sri Lanka. Ceylon Medical Journal 51, 89-92.

74. Allen A, Allen S, Rodrigo R et al. (2017) Iron status and anaemia in Sri Lankan secondary school children: a cross-sectional survey. PLOS ONE 12.

75. Shridevi KN, Rao RN, Madhavi P, Chandra Sekhar K, Deotale PG (2015) A study of nutritional status of adolescent girls of social welfare hostels in and around Vijayawada city. Indian J Public Health Res Dev 6, 40-46.

76. Jayatissa R, Ranbanda M. (2006) Prevalence of challenging nutritional problems among adolescents in Sri Lanka. Food Nutr Bull 27, 153-160.

77. Kawade R (2012) Zinc status and its association with the health of adolescents: a review of studies in India. Glob Health Action 5, 7353.

78. Gupta A, Kapil U, Ramakrishnan L, Pandey RM, Yadav Chander Pandey (2017) Prevalence of vitamin B12 and folate deficiency in school children residing at high altitude regions in India. Indian J Pediatr 84, 289-293.

79. Hettiarachchi M, Liyanage C, Wickremasinghe R, Hilmers DC, Abrahams SA (2006) Prevalence and severity of micronutrient deficiency: a cross-sectional study among adolescents in Sri Lanka. Asia Pac J Clin Nutr 15, 56-63.

80. Thoradeniya T, Wickremasinghe R, Ramanayake R, Atukorala S (2006) Low folic acid status and its association with anaemia in urban adolescent girls and women of childbearing age in Sri Lanka. BrJ Nutr 95, 511-516.

81. Kapil U, Sareen N (2014) Prevalence of ferritin, folate and vitamin B12 deficiencies amongst children in 5-18 years of age in Delhi. Indian J Pediatr 81, 312.

82. Kapil U, Pandey RM, Sharma B et al. (2018) Prevalence of vitamin D deficiency in children (6-18 years) residing in Kullu and 
Kangra Districts of Himachal Pradesh, India. Indian J Pediatr 85, 344-350.

83. Kapil U, Pandey RM, Goswami R et al. (2017) Prevalence of vitamin D deficiency and associated risk factors among children residing at high altitude in Shimla district, Himachal Pradesh, India. Indian J Endocrinol Metab 21, 178-183.

84. de Lanerolle-Dias M, de Silva A, Lanerolle P et al. (2012) Micronutrient status of female adolescent school dropouts. Ceylon Med J 57, 74-78.

85. Pandav CS, Mallik A, Anand K et al. (1997) Prevalence of iodine deficiency disorders among school children of Delhi. Natl MedJ India 10, 112-114.

86. Harun-Or-Rashid MK, Khatun UF, Yoshida Y, Morita S, Chowdhury N, Sakamoto J. (2009) Iron and iodine deficiencies among under-2 children, adolescent girls, and pregnant women of Bangladesh: association with common diseases. Nagoya J Med Sci 71, 39-49.

87. Agrawal VK, Agrawal P, Dharmendra (2013) Prevalence and determinants of xerophthalmia in rural children of Uttarpradesh, India. Nepal J Ophthalmol 5, 226-229.

88. Sinha A, Jonas JB, Kulkarni M, Nangia V (2011) Vitamin A deficiency in schoolchildren in urban central India: the central India children eye study. Arch Ophthalmol 129, 1095-1096.

89. Chakraborty S, Chopra M, Mani K et al. (2018) Prevalence of vitamin B-12 deficiency in healthy Indian school-going adolescents from rural and urban localities and its relationship with various anthropometric indices: a cross-sectional study. $J$ Hum Nutr Diet 31, 513-522.
90. Laxmaiah AA, Arlappa N, Balakrishna N et al. (2013) Prevalence and determinants of micronutrient deficiencies among rural children of eight states in India. Ann Nutr Metab 62, 231-241.

91. Selvarani P (2017) Prevalence of anaemia and its association with demographic factors among adolescent girls in Coimbatore district, India. Indian I Public Health Res Dev $\mathbf{8}$, 207-211.

92. Gupta AG (2018) Prevalence of iron deficiency anaemia in healthy adolescent girls from low to medium socio-economic strata living in one of the fastest developing economies. $\mathrm{BrJ}$ Haematol 181, 167-168.

93. Lamba R Jr, Misra SK, Agrawal R, Rana R Sr. (2014) To study the prevalence of anemia and related biosocial factors among college going adolescent girls in urban Agra. Indian J Public Health Res Dev 5, 35-38.

94. United Nations Children's Fund (2014) Global initiative on outof-school children: South Asia regional study covering Bangladesh, India, Pakistan and Sri Lanka. Kathmandu, Nepal.

95. Singh P, Gandhi S, Malhotra RK et al. (2020) Impact of using different growth references on interpretation of anthropometric parameters of children aged 8-15 years. Indian Pediatr 57, 124-128.

96. Paez A (2017) Grey literature: an important resource in systematic reviews. J Evid Based Med.

97. Moher D, Liberati A, Tetzlaff J et al. (2009) Preferred reporting items for systematic reviews and meta-analyses: the PRISMA statement. PLoS Med 6, e1000097. 